\title{
Burgund und das « unsichtbare Römische Reich » im Spiegel der sogenannten merowingischen Monetarmünzen. Eine Anmerkung
}

Jürgen Strothmann

\section{(2) OpenEdition}

1 Journals

\section{Édition électronique}

URL : https://journals.openedition.org/cem/14848

DOI : $10.4000 /$ cem. 14848

ISSN : 1954-3093

Éditeur

Centre d'études médiévales Saint-Germain d'Auxerre

Référence électronique

Jürgen Strothmann, «Burgund und das « unsichtbare Römische Reich » im Spiegel der sogenannten merowingischen Monetarmünzen. Eine Anmerkung », Bulletin du centre d'études médiévales d'Auxerre | BUCEMA [En ligne], 21.2 | 2017, mis en ligne le 07 février 201, consulté le 22 septembre 2022. URL : http://journals.openedition.org/cem/14848; DOI : https://doi.org/10.4000/cem.14848

Ce document a été généré automatiquement le 22 septembre 2022.

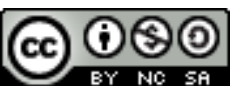

Creative Commons - Attribution - Pas d'Utilisation Commerciale - Partage dans les Mêmes Conditions 4.0 International - CC BY-NC-SA 4.0

https://creativecommons.org/licenses/by-nc-sa/4.0/ 


\title{
Burgund und das « unsichtbare Römische Reich » im Spiegel der sogenannten merowingischen Monetarmünzen. Eine Anmerkung ${ }^{1}$
}

\author{
Jürgen Strothmann
}

\section{NOTE DE L'ÉDITEUR}

Cet article fait référence à la carte 1 du dossier cartographique. Cette carte est réinsérée dans le corps du texte et le lien vers le dossier cartographique est donné en document annexe. 
1 Burgund ist im Frühmittelalter wohl nur als Bezeichnung für einen Raum verstehbar, der sich unmittelbar aus einem politischen Zusammenhang ergibt und auch nur über diesen definierbar ist. Als absolute geographisch fassbare Größe möchte ich es nicht definieren müssen. Denn die geographische Zugehörigkeit zum Reich der Burgunder ist zunächst nur als ein Ausdruck eben dieser politischen Gruppe greifbar ${ }^{2}$, wird dann im Reich der Merowinger zwar zunächst im Ganzen erhalten, um dann bereits im

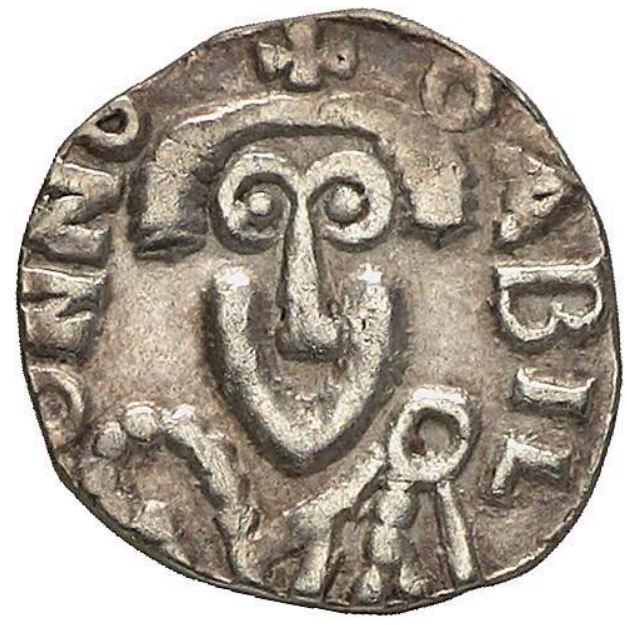
7. Jahrhundert keine geographische Integrität mehr aufzuweisen. An Burgund zeigt sich sehr deutlich, dass die fränkischen Teilreiche in der Zeit der Merowinger keine unmittelbare politische Qualität und schon gar keine geographische Integrität besitzen. Sie sind Ergebnisse eines beständigen nicht immer friedlichen - sozialen Aushandlungsprozesses unter den Eliten.

Die Teilreiche sind in der Merowingerzeit eben nicht die politischen Grundeinheiten, als die sie seit der Karolingerzeit gelten können. Das hat mit der politischen Überformung dieser sozialen Aushandlungsprozesse zu tun, die in der Karolingerzeit letztlich zu neuen Reichsbildungen führten.

Abb. 1a-b - Triens, Chalon-sur-Saône, 1,21 g, 14 mm
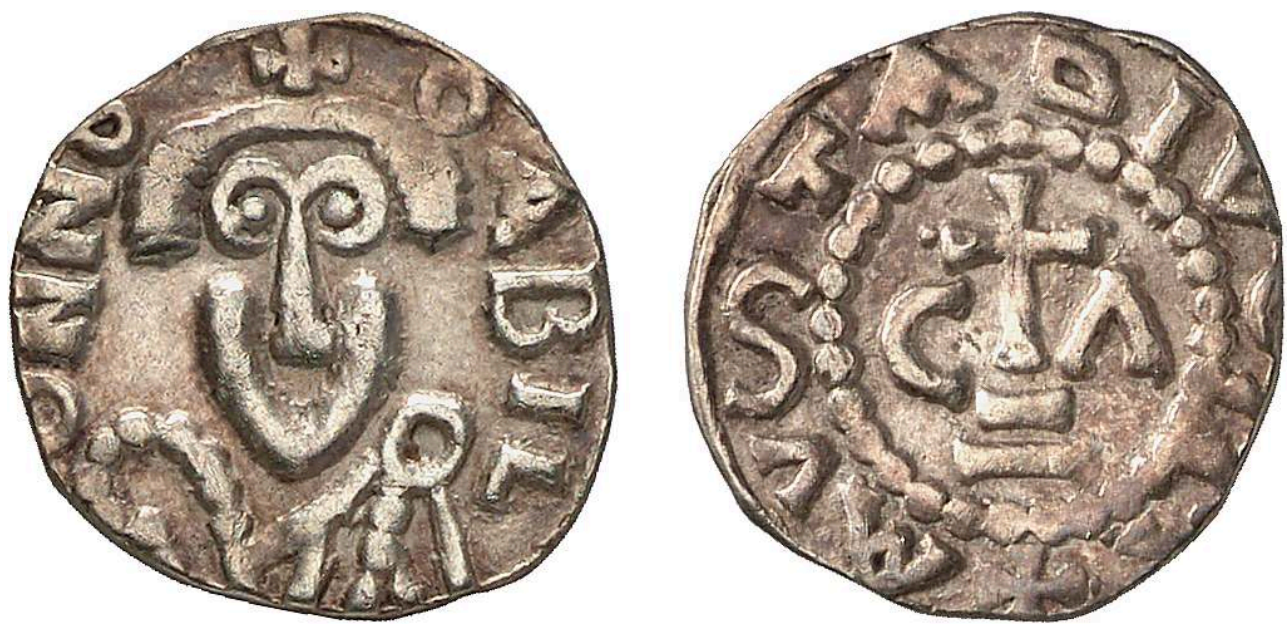

OABIL-ONNO / AVSTADIVS M, CA im Punktkreis für « Cabilonno ».

Münzkabinett der Staatlichen Museen zu Berlin, Objektnummer 18207480.

Abbildung und Beschreibung in K. DAHMEN und B. KLUGE, « Bestandskatalog der merowingischen Münzen des Münzkabinetts der Staatlichen Museen zu Berlin », in A. greule, J. JARNut, B. KLUge und M. SELIG (Hg.), Die merowingischen Monetarmünzen als interdisziplinär-mediaevistische Herausforderung, Paderborn, 2017, S. 155-292, Nr. 300, S. 234 / Tafel XIV (Foto von Lutz-Jürgen Lübke). 
Abb. 2a-b - Triens, Chalon-sur-Saône, 1,18 g, 12 mm
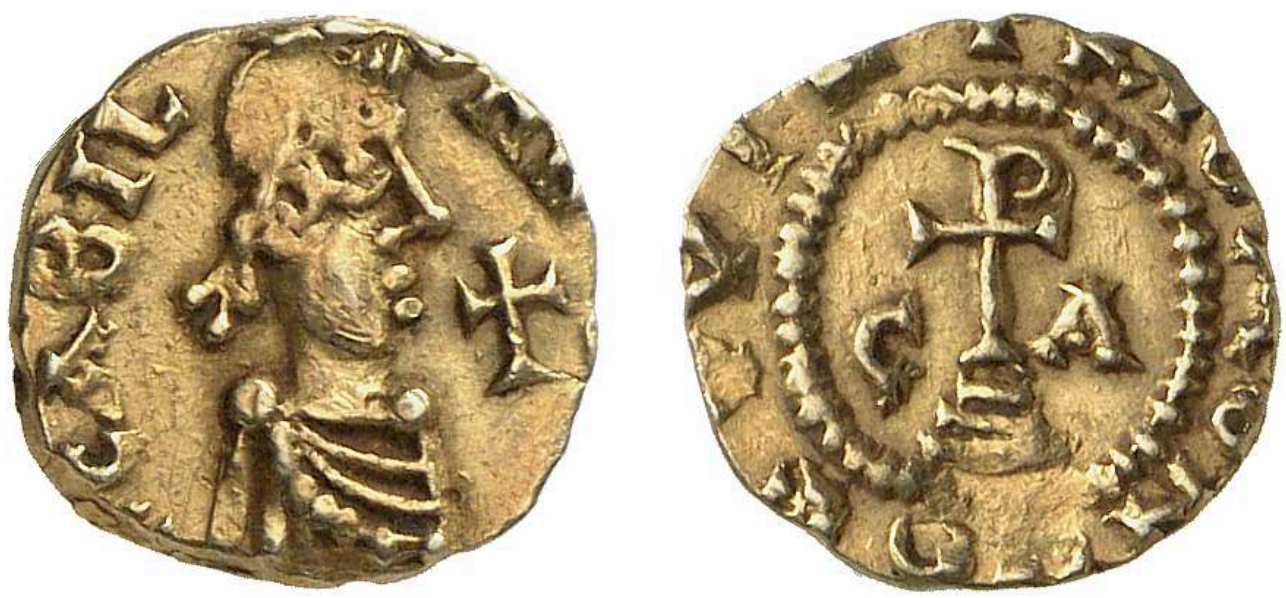

CABIL-ONN / VVINTRIO MON

Münzkabinett der Staatlichen Museen zu Berlin, Objektnummer 18202306.

Abbildung und Beschreibung in K. DAHMEN und B. KLUGE, «Bestandskatalog der merowingischen... », op. cit., Nr. 307, S. 237 / Tafel XIV (Foto von Lutz-Jürgen Lübke).

\section{Abb. 3a-b - Triens, Lausanne, 1,21 g, $13 \mathrm{~mm}$}
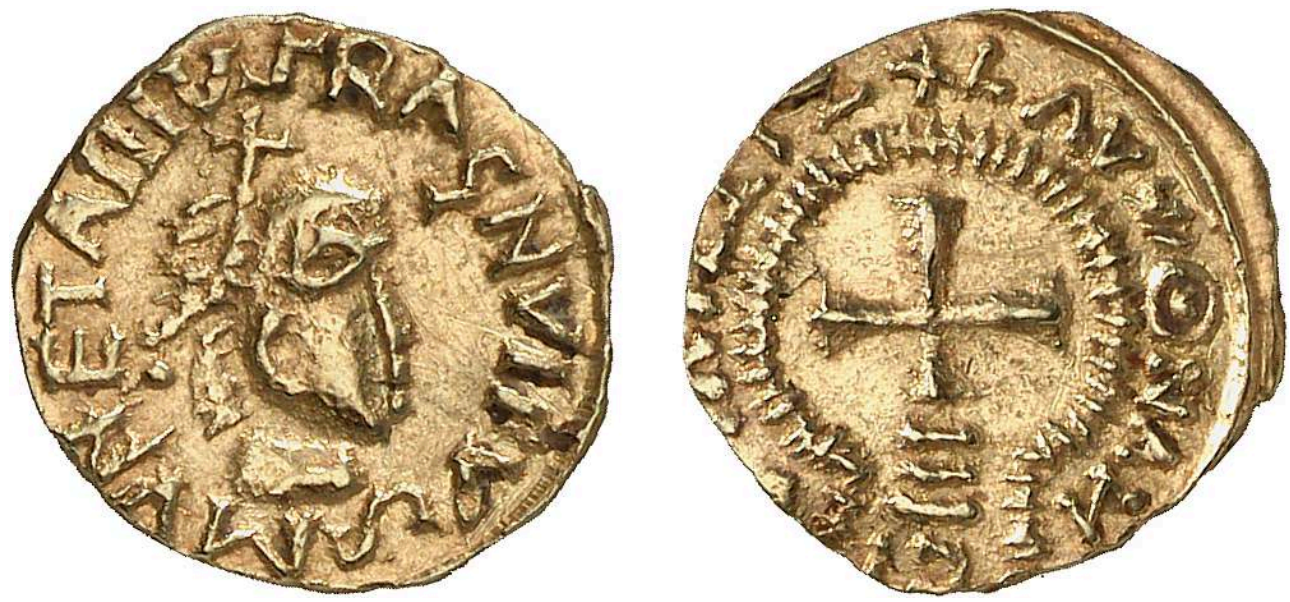

LAVSONAN - CI -V[E]TAI[..]IZ / RAGNVIFVS MVNETARIVS.

Münzkabinett der Staatlichen Museen zu Berlin, Objektnummer 18207494.

Abbildung und Beschreibung in K. DAHMEN und B. KLUGE, « Bestandskatalog der merowingischen... », op. cit., Nr. 315, S. 239 / Tafel XIV (Foto von Lutz-Jürgen Lübke). 
Abb. 4a-b - Triens, Sion (Valais), 1,22 g, $12 \mathrm{~mm}$
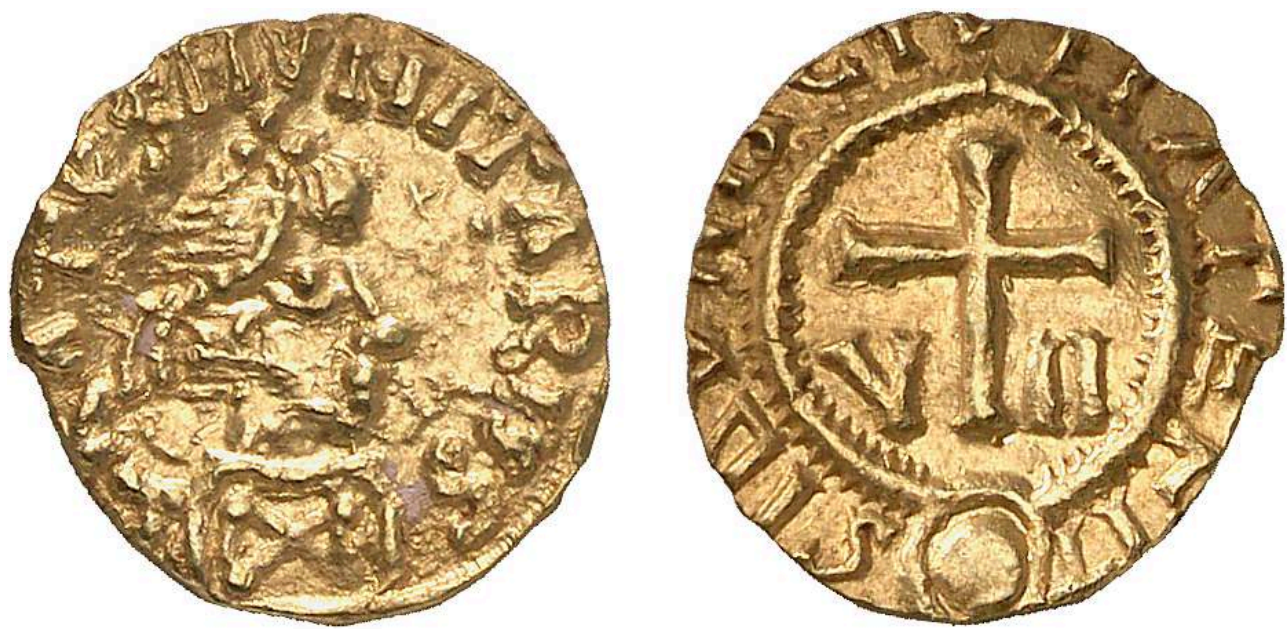

GRATVS MVNITARIVS / SIDVNIS CIVETATE FIT.

Münzkabinett der Staatlichen Museen zu Berlin, Objektnummer 18207526.

Abbildung und Beschreibung in K. DAHMEN und B. KLUGE, « Bestandskatalog der merowingischen... », op. cit., Nr. 326, S. 243 / Tafel XV (Foto von Lutz-Jürgen Lübke).

3 Die sogenannten "merowingischen Monetarmünzen ${ }^{3}$ zeigen für das 7. Jahrhundert sehr deutlich, dass die politischen und wirtschaftlichen Grundeinheiten Galliens in der Merowingerzeit die civitates waren, aus deren Bestand sich die Teilreiche zusammensetzten, die in einem ganz römischen Sinn als Provinzen aufgefasst wurden, nämlich als Aufgabenbereiche der Könige und damit als Verwaltungsbezirke ${ }^{4}$. Darauf gründet ein wesentliches Argument für die Annahme eines « unsichtbaren Römischen Reiches ", dass nämlich die Grundstruktur dieses Reiches im Westen alle völkerwanderungszeitlichen Stürme und auch die Reichsbildungen der Barbaren weitgehend unbeschadet überstanden hat. Unter dem Schlagwort des « unsichtbaren Römischen Reiches» lassen sich die Siegener Bemühungen fassen, ein auf den Erkenntnissen des Monetarmünzprojektes beruhendes Konzept zu verfolgen, nach dem die Kontinuität vom (sichtbaren) Römischen Reich im Westen zu den frühmittelalterlichen regna aus einer inneren kommunikativen Struktur unter den Eliten und der daraus folgenden politischen Ordnung von Kleinstaaten, den civitates nämlich, heraus zu verstehen ist. Diese Strukturen sind aufgrund ihrer kommunikativsozialen Basis mit dem Wegbrechen der römischen politischen Suprastruktur kaum substanziell in Mitleidenschaft gezogen worden. Das erklärt die Funktionsfähigkeit, die Gestalt und die maßgebliche kulturelle Orientierung der Nachfolgereiche des Römischen Reiches 5 . Schon das Römische Reich besteht aus den politischen und wirtschaftlichen Einheiten der civitates, die den Vorzug haben, dass ihre Politeia auf sozialen Grundbedingungen basiert und deshalb später unabhängig von der steuereintreibenden Behörde, ob Römer, Burgunder oder Franken, sich selbst erhält. Genau das lässt sich eben auch an den Merowingischen Monetarmünzen erkennen, deren Hauptfunktion in der Abgabenerhebung bestand und die mutmaßlich die Abgabenorte auf ihrem Revers nennen ${ }^{6}$, weshalb hier auch nicht von Prägeorten, sondern von Münzorten die Rede ist. Dieses Münzsystem nennt keine Teilreiche und lässt auch sonst nicht erkennen, dass das Frankenreich in Teilreiche gegliedert war. Lediglich regionale Unterschiede in der Ikonographie sind zu beobachten, die jedoch keine klaren Teilreichsgrenzen erkennen lassen. Das System funktioniert reichsweit bis 
zum Rhein und zeigt ein unmittelbares Verhältnis von Hauptorten und dem Gesamtreich. Unter den Orten befinden sich nahezu alle römischen civitas-Hauptorte, mehrere hundert pagus-Hauptorte und einige Klöster, Kirchen sowie Häfen und fisci, augenscheinlich alle Orte, von denen Abgaben irgendwelcher Art erhoben wurden.

Der von den Burgundern belegte geographische Raum bildet insofern eine bereits bestehende Größe, als dass er schon im Römischen Reich als eine Art "Wegeraum » verstehbar ist. Er verbindet die Rhône und damit Arles und das Mittelmeer über die Saône mit dem Rhein, der Seine und der Loire, so dass man durch Burgund muss, wenn man auf schnellem (Wasser-)Wege Nordgallien, das Rheinland und Britannien erreichen will. Außerdem gehören zu Burgund der westliche Hauptweg über die Alpen, nämlich über den großen St. Bernhard, den Poeninus, sowie die Wege über die Graischen Alpen nach Segusio (Susa). Nicht umsonst liegt das geistliche Zentrum des burgundischen Reiches am Weg vom St. Bernhard nach Gallien, in Acaunum, wo gegen Ende des 5. Jahrhunderts das dauerhaft bedeutende Kloster Saint-Maurice d'Agaune gegründet wurde ${ }^{7}$. So ist Burgund zwar nicht identisch mit antiken Provinzeinteilungen, die ja eine gewisse Willkürlichkeit auszeichnet, sondern basiert auf einer Kontinuität von civitates, also römischen Kleinstaaten, deren Hauptorte an den großen Straßen liegen. Unter ihnen befinden sich mehrere ausgesprochen bedeutende römische Städte, etwa Lyon und Vienne.

Burgund ist aber nicht nur das Herrschaftsgebiet und der Kommunikationsraum der gens burgundionum, sondern durch seine Einrichtung seit den 40er Jahren des 5. Jahrhunderts ein gallischer Teilraum, der lange vor der territorialen Hegemonie der Franken in Gallien politische Strukturen aus dem noch bestehenden römischen Westreich unmittelbar übernahm ${ }^{8}$ und damit mutmaßlich einen Zustand der sozialen und institutionellen sowie politischen Ordnungen teilweise einfror, der eben nicht als eigentlich poströmisch bzw. nachantik einzuschätzen ist. Sollte diese Vermutung zutreffend sein, hieße dies, dass das ehemalige Reich der Burgunder in Gallien einen besonderen Kontinuitätsraum geschaffen haben würde, der bei der reibungslosen Übernahme durch die Franken als ein der antiken Ordnung sehr nahestehender Raum anzusehen ist.

6 Das lässt sich überprüfen, nämlich anhand der «Merowingischen Monetarmünzen », die die politische und wirtschaftliche Substruktur Galliens im 7. Jahrhundert abbilden. Im Laufe der römischen Kaiserzeit und mutmaßlich auch danach noch wird die Integrität der ursprünglichen politischen Subsysteme, der civitates nämlich, beschädigt, indem aus ihnen neue politische Subsysteme herausgelöst werden. Das scheint ein kontinuierlicher Prozess zu sein, der im Wesentlichen der Effizienz von Abgabenerhebung und politischer Kontrolle geschuldet ist.

Die Münzen tragen allesamt Ortsnamen, oft mit Qualifikationen ihres politischen bzw. wirtschaftlichen Status, etwa als civitas-Hauptort (s. Abb. 4), als pagus-Hauptort oder als basilica oder als pons. Wenn im Raum Burgund die Zahl der neben den civitates in der Münzprägung sichtbaren Orte deutlich niedriger sein sollte als in anderen wirtschaftlich prosperierenden Regionen Galliens, müsste man daraus schließen, dass es entweder weniger solcher sekundär bedeutender Orte gegeben haben müsste oder aber diese tatsächlich noch Teil der civitates waren. Letzteres lässt sich für die Provence vermuten, in der sogar mehrere civitates wohl unter dem Münzort Marseille figurieren ${ }^{10}$. Beides spräche dafür, dass eine wahrscheinliche weitere Auflösung der 
civitas-Integrität in den letzten Jahrzehnten römischer Herrschaft in Burgund nicht mehr stattgefunden haben würde.

Leider fehlt aktuell ein präzises Instrument, mit dem man dieses Phänomen sicher feststellen könnte, denn die numismatischen corpora der Merowingischen Monetarmünzen leiden allesamt an einer gewissen Intransparenz, was die Identifizierung von Orten und damit ihre Lokalisierung betrifft. Das auf Vollständigkeit ausgelegte Werk von De Belfort enthält zwar eine große Zahl an erfassten Münzen, zugleich aber auch Dubletten; manche Verzeichnungen sind nicht mehr überprüfbar ${ }^{11}$. Die Arbeit von Prou beschränkt sich auf den Bestand der Bibliothèque nationale de France ${ }^{12}$, der immerhin der größte und zudem seit dem Erscheinen des Katalogs auch um einiges gewachsen ist. Obwohl die große sprachwissenschaftliche Untersuchung von Egon Felder die Neuzugänge berücksichtigt und auch die Münzorte weitgehend lokalisiert, ist sie doch, weil es dem Autor vornehmlich um die Personennamen geht, ebenfalls in den Lokalisierungen nicht ausreichend transparent ${ }^{13}$. Es gibt zwar von Depeyrot einen mehrbändigen Ersatz für die Arbeit von De Belfort, aber auch hier finden sich in besonderem Maße Intransparenzen, die Lokalisierungen sind z.T. eigenwillig und entsprechen mitunter nicht den Anforderungen der Sprachwissenschaften ${ }^{14}$.

9 Überhaupt sind Lokalisierungen nur sinnvoll im Verbund mit den Sprachwissenschaften $\mathrm{zu}$ leisten. Es müssten dabei numismatische, sprachwissenschaftliche und rein geschichtswissenschaftliche Kriterien mit- und gegeneinander abgewogen werden. Und dennoch war es in einem interdisziplinären von der Deutschen Forschungsgemeinschaft geförderten Projekt zu den "merowingischen Monetarmünzen als interdisziplinäre Herausforderung », das vor allem den Personennamen galt, nicht möglich, die Zahl der sicher lokalisierten Münzorte signifikant zu erhöhen ${ }^{15}$. Wollte man eine wissenschaftlich brauchbare Karte zeichnen oder auch nur die Orte in einem Raum sicher feststellen, ginge dies nur mit einem System, in dem Unsicherheiten in der Identifizierung der Orte nach Grad der Unsicherheit ausgewiesen wären oder aber zumindest ein Bestand von Namen verhältnismäßig sicher identifizierter Orte manifest wird. In der Tat gibt es eine Datenbank, die zumindest dem zweitgenannten Prinzip folgt, die aber bisher nur die Daten der Münzbestände der Bibliothèque nationale de France und des Münzkabinetts der Staatlichen Museen zu Berlin enthält und deshalb ebenfalls noch nicht für eine auch nur annähernd präzise Feststellung der Zahl von Ortsnamen für einen bestimmten Raum nutzbar ist ${ }^{16}$.

10 Ein Survey auf der Basis des Katalogs von Depeyrot hat ergeben, dass in der Tat signifikante Unterschiede zwischen den verschiedenen Regionen bestehen. Depeyrot gliedert anders als Prou nach Departements, was den Nachteil hat, dass eben nicht die absolute Zahl von Münzorten in einer civitas erfassbar ist. Das wird vermutlich auch nicht möglich sein, weil die antiken civitas-Grenzen nicht unbedingt mit den modernen Bistumsgrenzen deckungsgleich sein müssen. Verglichen wurde Burgund mit dem Maas-Moselraum und Zentralfrankreich um Limoges.

11 Burgund weist eine Münzortdichte von im Durchschnitt 4,3 auf ein Departement auf mit den Schweizer Kantonen -, Zentralfrankreich von 11,5 und der Maas-Mosel-Raum von 10. Das scheint durchaus weitere Untersuchungen zu rechtfertigen. Während das Departement Saône-et-Loire in Burgund mit 9-11 Münzorten - allein drei Münzorte sind unmittelbar mit dem civitas-Hauptort Chalon-sur-Saône verbunden (Cabillonum, 
vgl. Karte 1 und Abb. 1-2), nämlich ein palatium, die civitas und Saint-Marcel - die meisten solcher Orte aufweist und dabei drei civitas-Hauptorte beherbergt, nämlich Autun und Chalon-sur-Saône sowie Mâcon, das jedoch in der spätantiken « Notitia Galliarum » noch als castrum figuriert, finden sich im Departement Indre-et-Loire mit dem civitas-Hauptort Tours sogar 33 Münzorte. Burgund kommt mit 13 civitasHauptorten auf 52 Münzorte, der Raum im Nordwesten (Maas-Mosel) kommt mit 7 civitas-Hauptorten auf 70 Münzorte. In Zentralfrankreich sind 5 civitas-Hauptorte unter 143 Münzorten, dies in 11 Departements.

Karte 1 - Die Provinz Maxima Sequanorum in der Spätantike (um 395 u. Z.)

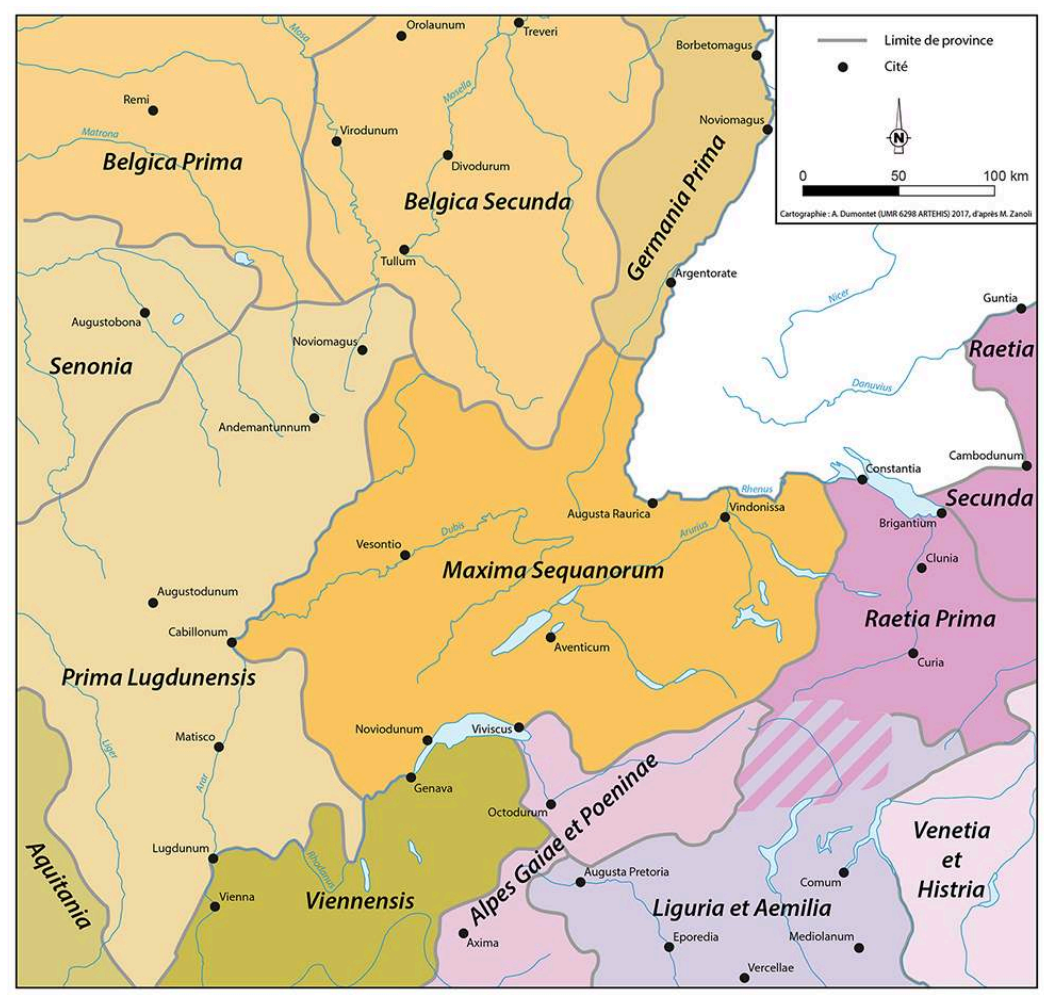

12 Bernd Kluge hat in einer umfassenden Untersuchung der Prägeintensität $\mathrm{zu}$ allen bekannten Münzorten ein Punktesystem entwickelt, das sowohl die bekannte Stempelzahl als auch die bekannten Münzen selbst in ihrer Menge bewertet und dabei nach Gold- und Silberprägungen differenziert ${ }^{17}$. Bei den Goldprägungen steht Marseille an der Spitze der Münzorte mit 694 Punkten. Dies ist in besonderer Weise dem Mittelmeerhandel geschuldet. Außerdem scheint Marseille als Münzhauptort der Provence zu fungieren ${ }^{18}$. Unmittelbar darauf folgt Chalon-sur-Saône mit 587 Punkten. Ähnlich wie Marseille scheint Chalon-sur-Saône in besonderer Weise eine zentralörtliche Funktion zuzukommen. An neunter Stelle steht Lyon mit 206 Punkten. Aber auch Autun (153), Sion (152) und Vienne (116) zählt Kluge unter die "großen Münzstätten " (Abb.4). Lausanne (44) und Genf (46) sortiert Kluge immerhin noch unter die «mittelgroßen Münzstätten» (Abb.3). Das korrespondiert mit dem dargelegten Befund zu der Zahl der Münzorte insofern, als dass diese Zahlen nahelegen, dass die civitas-Hauptorte offensichtlich der Prägemenge nach fehlende weitere Münzorte in ihrem (ehemaligen) Territorium kompensieren. Das könnte man im Einzelnen zusammenrechnen und vergleichen. Da wir aber nicht genug über die Relation der Regionen im Hinblick auf ihre absolute Wirtschaftskraft wissen, würde der 
Aussagewert des Ergebnisses gering sein. Es mag genügen, dass im Falle von Chalonsur-Saône jedenfalls die Prägemenge so hoch ist, dass es nur mit Marseille überhaupt vergleichbar ist, in dessen Umgebung sich mutmaßlich keine kleineren Münzorte befinden. Die nächst geringeren Münzorte sind Banassac mit 438 Punkten und Clermont-Ferrand mit 314 Punkten. Alle anderen Münzorte in Gallien liegen unter 300 Punkten.

Vorbehaltlich einer Untersuchung der Münzortdichte im Verhältnis zu antiken politischen Strukturen und damit vor allem den civitates und ihren Hauptorten, die jeweils auch die Lokalisierungen nach dem Forschungsstand bewertet, lässt sich aber doch feststellen, dass Burgund ein Raum ist, in dem die Zerstückelung der politischen bzw. wirtschaftlichen Landschaft augenscheinlich nicht in dieser Weise vonstatten ging, wie in anderen Regionen Galliens. Im Maas-Mosel-Raum liegt dies mutmaßlich an der besonders exponierten Lage gegenüber den einfallenden Barbaren und an der wirtschaftlichen Prosperität der Seille-Region (dép. Moselle), die nicht unwesentlich vom Salz lebte. In Zentralfrankreich mit einer mittleren Münzortdichte pro Departement von über 10 lässt sich zunächst vermuten, dass eben dies - wie vermutlich auch im Norden - an einer längeren Phase ohne konsolidierte civitas-übergreifende territoriale Herrschaft liegen wird ${ }^{19}$, wie eben der Blick auf Burgund nahelegt, das vermutlich kontinuierlich eine solche civitas-übergreifende Herrschaft kannte ${ }^{20}$.

Reçu : 27 juillet 2017 - Accepté : 4 novembre 2017

\section{ANNEXES}

Carte 1 - La province Maxima Sequanorum dans l'Antiquité tardive (vers 395)

\section{NOTES}

1. Der Beitrag sei Jörg Jarnut gewidmet, in Dankbarkeit, auch für intensive Gespräche über ein « unsichtbares Römisches Reich » als Konzept für den Epochenwandel. Karsten Dahmen und dem Münzkabinett der Staatlichen Museen zu Berlin danke ich für die Abbildungen.

2. S. zur burgundischen Sprache und dem ihr zuzuordnenbaren Raum W. HAUBRICHS, «Sprachliche Integration, Sprachinseln und Sprachgrenzbildung im Bereich der östlichen Gallia. Das Beispiel der Burgunden und der Franken", in T. KöLZER und R. SCHIEFFER (Hg.), Von der Spätantike zum Frühen Mittelalter: Kontinuitäten und Brüche, Konzeptionen und Befunde, Ostfildern, 2009, S. 61-100 ; ID., "Les Chamaves et les autres: une enquête linguistique sur les traces des Chamaves, Hattuaires, Varasques, Scotinges et Burgondes au nord de la Bourgogne », http:// journals.openedition.org/cem/14779. S. zu den methodischen Implikationen, v. a. zur Integration von Räumen und Personen C. EHLERS, Rechtsräume. Ordnungsmuster im Europa des frühen Mittelalters, Berlin/Boston, 2016, S. 102.

3. Zwischen etwa 585 und 670 wurden in Gallien ausschließlich Trienten geprägt, Drittelsolidi, die die weitaus stärker an den byzantinischen Prägungen orientierten "pseudoimperialen » Solidi des 6. Jahrhunderts ablösten und die selbst nahezu schlagartig in eigentlich 
mittelalterliche Denarprägungen übergingen, nachdem bereits über Jahrzehnte eine vermutlich kontinuierliche Verringerung des Feingehaltes vorgenommen worden war. Diese Münzprägung kann unterteilt werden in solche mit Königsnamen und solche ohne, wobei die allermeisten Prägungen nur einen oder zwei Monetare nennen und die « königlichen » Prägungen auf wenige Münzorte beschränkt sind. Die Gestaltung ist insofern einheitlich, als dass die «Monetarmünzen » alle einen Ortsnamen und mindestens einen Personennamen mitteilen, sie in aller Regel ein Herrscherbild auf der Vorderseite führen, das zwar als Kaiserbild gestaltet ist, aber durchaus auch für die Königsherrschaft stehen kann, und sie auf der Rückseite in der Regel ein Kreuz tragen, oft auf mehreren Stufen. Hinzu kommen häufig Ortsqualifikationen, wie CIVITAS und VICVS. Gelegentlich kommt noch ein Kürzel hinzu, das auf einen Hauptort verweist. S. die Münzkommentare von K. DAHMEN und B.KLUGE, «Bestandskatalog der merowingischen Münzen des Münzkabinetts der Staatlichen Museen zu Berlin », in A. GREULE, J. JARNUT, B. KLUGE und M. SELIG (Hg.), Die merowingischen Monetarmünzen als interdisziplinär-mediaevistische Herausforderung, Paderborn, 2017, S. 155-292, und die Abbildungen auf den Tafeln ebenda. Der Katalog ist auch online verfügbar : http://ikmk.smb.museum/home?lang=de-. S. auch P. GRIERSON und M. BLACKBURN, The Early Middle Ages, 5th - 10th centuries, Cambridge, 1986.

4. J. STROTHMANN, "Merowingische Monetarmünzen und die Gallia im 7. Jahrhundert", in A. GREULE u. a. (Hg.), Merowingische Monetarmünzen..., op.cit., S.11-69, hier S.56. Unsere Erkenntnisse basieren auf einem von der Deutschen Forschungsgemeinschaft in den Jahren 2007-2009 geförderten interdisziplinären Projekt zum besseren Verständnis der Merowingischen Monetarmünzen und vor allem der auf ihnen in großer Zahl mitgeteilten Personennamen.

5. S. dazu J. strothmanN, «The Evidence of Numismatics. Merovingian Coinage and the Place of Frankish Gaul and its Cities in an "invisible" Roman Empire ", in B. EFFROS und I. MOREIRA (Hg.), The Oxford Handbook of the Merovingian World, Oxford [im Druck]. S. programmatisch J. STROTHMANN, «Das "unsichtbare römische Reich". Zum Fortbestehen eines Raumes über seine Todesanzeige hinaus », in C. EHLERS (Hg.), Rechtsräume [im Druck].

6. J. StRothmAnN, «Königsherrschaft oder nachantike Staatlichkeit? Merowingische Monetarmünzen als Quelle für die politische Ordnung des Frankenreiches », Millennium, 5 (2008), S.353-381, hier S.366f. S. auch ID., «Das 7.Jahrhundert in neuem Licht. Merowingische Monetarmünzen als Quelle für eine nachantike Gesellschaftsordnung Galliens », Nouvelle revue d'onomastique, 54 (2012), S. 89-110; s. aber auch schon I. H. GARIPZANOV, « The Coinage of Tours in the Merovingian period and the Pirenne thesis", Revue belge de numismatique, 147 (2001), S. 79-118 und dezidiert zur Münzprägung im Zusammenhang mit der Steuererhebung in Limoges J.-F. BOYER, «À propos des triens mérovingiens : approche du système de collecte et de traitement de la recette fiscale en Limousin aux VI $-\mathrm{VII}^{\mathrm{e}}$ siècles ", Annales du Midi, 119 (2007), S. 141-157. Zur Deutung der Ortsnamen als Abgabenerhebungsorte s. auch schon A. M. STAHL, The Merovingian coinage of the region of Metz, Louvain-la-Neuve, 1982. Vgl. auch K. DAHMEN, «Zum Münzwesen des Merowingerreiches ", in A. GREULE u. a. (Hg.), Merowingische Monetarmünzen..., op. cit., S. 71-124 ; und mit einer eigenen Deutung des Systems B. KLUGE, «Die merowingischen Monetarmünzen : Epochenwandel im Münzwesen - Münzwesen im Epochenwandel. Numismatische Handreichungen für Historiker", in J. JARNUT und J. STROTHMANN (Hg.), Die Merowingischen Monetarmünzen als Quelle zum Verständnis des 7. Jahrhunderts in Gallien, Paderborn, 2013, S. 33-92. M. F. HENDY, «From Public to Private: The Western Barbarian Coinages as a Mirror of the Disintegration of Late Roman State Structures », Viator, 19 (1988), S. 29-78.

7. M. ZUFFEREY, Die Abtei Saint-Maurice d'Agaune im Hochmittelalter (830-1258), Göttingen, 1988, S. $30 \mathrm{ff}$.

8. W. HAUBRICHS, "Ein namhaftes Volk - Burgundische Namen und Sprache des 5. und 6. Jahrhunderts ", in V. GALLÉ (Hg.), Die Burgunder. Ethnogenese und Assimilation eines Volkes, Worms 2008, S. 154f. sieht im burgundischen regnum « eine von Romanen maßgeblich mitgestaltete 
Misch-Gesellschaft ». S. zu einer solchen sehr frühen Akkulturation auch I. wood, « Gentes, Kings and Kingdoms - The Emergence of States: The Kingdom of the Gibichungs ", in H.-W. GOETZ, J. JARNUT und W. POHL (Hg.), Regna and Gentes. The Relationship between Late Antique and Early Medieval Peoples and Kingdoms in the Transformation of the Roman World, Leiden/Boston, 2003, S. 243-269, S. $262 \mathrm{f}$.

9. J. StRothmann, «Civitas-Hauptorte und ihre Benennungen als Quelle für den Wandel der politischen Struktur Galliens bis zum 8. Jahrhundert », in J. JARNUT und J. STROTHMANN (Hg.), Die Merowingischen Monetarmünzen..., op. cit., S. 613-628, hier S.617. S. zu den civitates und ihrem Wandel v. a. C. WITSCHEL, «Die spätantiken Städte Galliens : Transformationen von Stadtbildern als Ausdruck einer gewandelten Identität », in S. DIEFENBACH und G. M. MÜLLER (Hg.), Gallien in Spätantike und Frühmittelalter. Kulturgeschichte einer Region, Berlin/Boston, 2013, S. 153-200.

10. S. dazu den Ortskommentar zu Marseille bei J. STROTHMAnN, "Kommentare zu einigen Hauptorten ", in J. JARNUT und J. STROTHMANN (Hg.), Die Merowingischen Monetarmünzen..., ibid., S. 403-446, hier S. $420 f$. S. zu Marseille v. a. S. T. LOSEBY, "Marseille and the Pirenne Thesis, I: Gregory of Tours, the Merovingian kings and "un grand port" ", in R. HODGES und W. BOWDEN (Hg.), The Sixth Century. Production, Distribution and Demand, Leiden, 1998, S. 203-229 und ID., "Marseille and the Pirenne Thesis, II : "Ville Morte" ", in I. L. HANSEN und C. WicKHAM (Hg.), The Long Eighth Century, Leiden/Boston/Köln, 2000, S. 167-193.

11. A. DE BELFORT, Description générale des monnaies Mérovingiennes, Bd. I-IV, Paris, 1892-1894.

12. M. PROU, Catalogue des monnaies françaises de la Bibliothèque Nationale. Les monnaies mérovingiennes, Paris, 1892.

13. E. FELDER, Die Personennamen auf den merowingischen Münzen der Bibliothèque nationale de France, München, 2003.

14. G. DEPEYROT, Le numéraire mérovingien. L'âge de l'or, Bd. 1-4, Wetteren, 1998.

15. Vg. Anm. 2. S. nun den v. a. sprachwissenschaftlichen Versuch von R. EUFE, Die Ortsnamen auf den merowingischen Monetarmünzen des Münzkabinetts der Staatlichen Museen Berlin, Berlin/New York, elektronische Vorabversion: http://nbn-resolving.de/urn/resolver.pl? urn=urn:nbn:de:bvb:355-epub-309258 (zuletzt 31. 5. 2017). Zu den Ortsnamen der Auvergne s. J.P. СнАмвоN, « L'identification des noms d'ateliers monétaires mérovingiens (Arvernie et entours) : point de vue de linguiste ", Revue numismatique, 2001, S. 347-405.

16. Die Datenbank wird der Datenbank von «Nomen et Gens » angeheftet werden : http:// www.neg.uni-tuebingen.de/?q=de/datenbank (zuletzt 31. 5. 2017).

17. B. KLUGE, "Die merowingischen Monetarmünzen », in J. JARNUT und J. STROTHMANN (Hg.), Die Merowingischen Monetarmünzen..., op. cit., S. 33-92, S. 66ff.

18. J. STROTHMANN, « Kommentare zu einigen Hauptorten », in A. GREULE u. a. (Hg.), Merowingische Monetarmünzen..., op. cit., S. 403-446, S. 420 « MASILIA ».

19. S. die Annahme von J. StrothmanN, «Civitas-Hauptorte...", op. cit., auf der Basis des Ortsnamenwandels. Über das Maß der inneren Ordnung der frühen regna im Westen müsste man folglich genauer nachdenken.

20. S. zu dem Prozess der Aneignung des Raumes durch die Burgunden I. wOOD, «Gentes, Kings and Kingdoms - The Emergence of States: The Kingdom of the Gibichungs ", in H.-W. GOETZ, J. JARNUT und W. POHL (Hg.), Regna and Gentes..., op. cit., S. 243-269, hier S. $259 f f$. 


\section{RÉSUMÉS}

La Bourgogne n'a jamais eu le statut de province romaine, mais elle représente un espace économique, qui, pendant le haut Moyen Âge, prend une certaine importance territoriale, et ceci n'est pas seulement dû à la domination par les Burgondes. En raison de la prise de pouvoir des Burgondes, qui a eu lieu relativement tôt et de façon organisée, le processus de désintégration des structures politiques a été ici interrompu plus tôt qu'ailleurs en Gaule. Ce constat est illustré par la situation monétaire du $\mathrm{VII}^{\mathrm{e}}$ siècle en Bourgogne, où l'on trouve bien moins d'ateliers de frappe en dehors des chefs-lieux de cités que dans les autres régions de la Gaule. Cela semble indiquer une très forte continuité du monde romain en Bourgogne, tant sur le plan monétaire que dans d'autres domaines.

The Burgundians became part of the Roman Empire before it collapsed as a political system in the West. Therefore, in terms of civilisation and political organisation Burgundy appears to have been more Roman than other parts of Gaul. This can be demonstrated by a survey of the mints in the different regions and a comparison of their number. In Burgundy, most of the places of lower rank were not mentioned on the coins. That's why we can assume that the fragmentation of the city-states did not go as far as in other Gaulish regions, where the cities had remained independent for some time after the end of the Western Roman Empire.

\section{INDEX}

Mots-clés : cité, empire romain, Gaule mérovingienne, monnaies mérovingiennes, état, administration

Schlüsselwörter : civitas, römisches Reich, merowingisches Gallien, Monetarmünzen, Verwaltung

\section{AUTEUR}

\section{JÜRGEN STROTHMANN}

Historisches Seminar, Université de Siegen 